
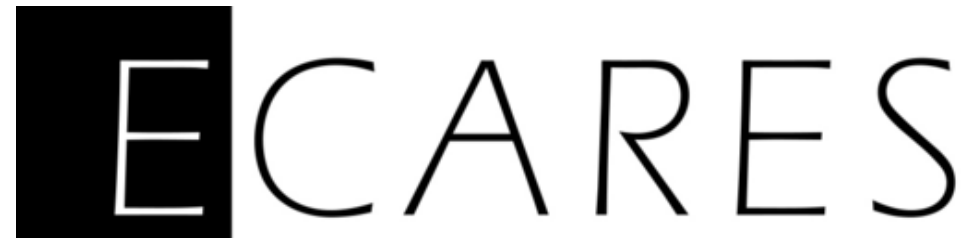

\title{
A Note on Estimating Wishart Autoregressive Model
}

Roxana Halbleib

SBS-EM, ECARES, Université Libre de Bruxelles and CoFE

ECARES working paper 2010-043

Konstanzer Online-Publikations-System (KOPS)

URL: http://nbn-resolving.de/urn:nbn:de:bsz:352-290081

\section{ECARES}

ULB - CP 114

50, F.D. Roosevelt Ave., B-1050 Brussels BELGIUM www.ecares.org 


\title{
A Note on Estimating Wishart Autoregressive Model
}

\author{
Roxana Halbleib*广 \\ Université libre de Bruxelles, ECARES, CoFE
}

\begin{abstract}
This note solves the puzzle of estimating degenerate Wishart Autoregressive processes, introduced by Gourieroux, Jasiak and Sufana (2009) to model multivariate stochastic volatility. It derives the asymptotic and empirical properties of the Method of Moment estimator of the Wishart degrees of freedom subject to different stationarity assumptions and specific distributional settings of the underlying processes.
\end{abstract}

JEL classification: $C 32, C 46, C 51$

Keywords: Wishart autoregressive process, asymptotic properties, realized covariance, log-normal distribution.

\section{Introduction}

Recently, Gourieroux et al. (2009) have proposed a new dynamic model of time series of multivariate volatilities, called Wishart Autoregressive (WAR) model. The

*European Center for Advanced Research in Economics and Statistics (ECARES), Université libre de Bruxelles, Solvay Brussels School of Economics and Management, Avenue F. Roosevelt, 50, CP 114, B1050 Brussels, Belgium. Phone + 322650 3469, email: Roxana.Halbleib@ulb.ac.be. The author gratefully acknowledge financial support from the Belgian National Bank and the IAP P6/07 contract, from the IAP programme (Belgian Scientific Policy), 'Economic policy and finance in the global economy'. The author is a member of ECORE, the recently created association between CORE and ECARES. A previous extended version of this note circulated under the title "Properties of the Realized Volatility Wishart Autoregressive Model" and under the maiden name of the author, Roxana Chiriac.

${ }^{\dagger}$ The paper was written while the author was research fellow at Department of Economics and Center of Finance and Econometrics (CoFE) at the University of Konstanz, Germany. Financial support from the German Science Foundation within the project "Price-, Liquidity- and Credit Risks: Measurement and Allocation" is gratefully acknowledged. 
model is based on the Wishart distribution of sample variance-covariance matrices computed from i.i.d. vectors of autoregressive Gaussian processes. One important property of the model, is that the number of Gaussian processes, which gives the number of the degrees of freedom (d.f.) of the Wishart distribution, has to be larger than the dimension of the matrix process in order to describe a non-degenerate WAR process for the underlying data. However, empirical results from applying the model to series of realized covariance matrices of stock returns reveal degenerate Wishart distributions of the underlying covariance matrix series. In this note we show that inconsistencies between the model assumptions and the statistical properties of the underlying data may lead to such results. We derive the properties of the Method of Moment (MM) estimator of the Wishart d.f., highlighted by Gourieroux et al. (2009), under different stationarity settings and we show that nonstationary (cointegrated) autoregressive Gaussian processes offer the appropriate theoretical setting for describing the empirical results (Section 3). Moreover, we show that violations of the distributional assumptions of the underlying data severely affect the estimation results and the interpretation of the model (Section 4).

\section{Preliminaries}

Let $x_{k, t}$ with $k=1, \ldots, K$ be independent processes of dimension $n \times 1$ given by:

$$
x_{k, t}=M x_{k, t-1}+\varepsilon_{k, t} \quad \varepsilon_{k, t} \stackrel{\text { iid }}{\sim} \mathrm{N}(0, \Sigma), \quad k=1, \ldots, K
$$

Gourieroux et al. (2009) define the process $Y_{t}$ given by:

$$
Y_{t}=\sum_{k=1}^{K} x_{k, t} x_{k, t}^{\prime},
$$

to be a Wishart Autoregressive process of order 1, WAR(1), dimension $n, K$ d.f., latent autoregressive matrix $M$ and innovation covariance matrix $\Sigma$. An important property of the Wishart distribution is that the matrices $Y_{t}$ are positive definite, if and only if $K \geq n$. Whenever $K<n$, the process $Y_{t}$ consists of a sequence of singular covariance matrices with degenerate Wishart distribution. 


\section{Asymptotic properties of WAR(1)}

Based on a sequence of covariance matrices $Y_{1}, \ldots, Y_{t}$, Gourieroux et al. (2009) derive the MM estimator of $\mathrm{K}$ to be:

$$
\hat{K}=\frac{2\left[\alpha^{\prime} \hat{\Sigma}^{*}(\infty) \alpha\right]^{2}}{\hat{V}\left[\alpha^{\prime} Y_{t} \alpha\right]}
$$

where $\alpha$ is of dimension $n \times 1$ and is a interpreted as a portfolio allocation and $\hat{\Sigma}^{*}(\infty)$ is a solution to $\hat{\Sigma}^{*}(\infty)=\hat{M} \hat{\Sigma}^{*}(\infty) \hat{M}^{\prime}+\hat{\Sigma}^{*}$, where $M$ and $\Sigma^{*}$ are estimated by the first-order Method of Moments. In what follows we derive the asymptotic properties of this estimator subject to different stationarity assumptions of the latent autoregressive Gaussian processes.

Proposition 3.1: If $x_{k t}$ are stationary $(k=1, \ldots, K)$, then the MM estimator of the Wishart d.f. is consistent and asymptotically normal distributed with:

$$
\sqrt{T}(\hat{K}-K) \stackrel{d}{\rightarrow} N\left(0, \sum_{j=-\infty}^{\infty} \gamma_{j}\right),
$$

where $\gamma_{j}=\mathrm{E}\left[\left(S_{t}-K\right)\left(S_{t-j}-K\right)\right]$ with $S_{t}=\sum_{k=1}^{K} \frac{\left(\alpha^{\prime} x_{k, t}\right)^{2}}{\alpha^{\prime} \Sigma(\infty) \alpha}$ and $\Sigma(\infty) \equiv V\left[x_{k, t}\right]$.

Proof. See Appendix A.1.

From Proposition 3.1, we can conclude that the stationarity assumption is sufficient for obtaining consistent estimators of the Wishart d.f. Thus whenever $K>n$, then (asymptotically) $\hat{K}>n$ and $\hat{K}$ describes a non-degenerate Wishart process for the underlying covariance matrix process. But is the stationarity assumption also necessary? We answer this question by relaxing this assumption and by considering latent autoregressive processes with unit roots.

Proposition 3.2: If $x_{k t}$ have unit root $(k=1, \ldots, K)$, then the MM estimator of the Wishart d.f. is inconsistent and:

$$
\hat{K} \stackrel{d}{\rightarrow} K \int_{0}^{1}[W(s)]^{2} d s .
$$

where $W(\cdot)$ stands for the standard Brownian motion. 
Proof. See Appendix A.2.

Even though one may assume that $K$ is larger than $n$, the WAR(1) process is not guaranteed to be non-degenerate, given that $\hat{K}$ might be (asymptotically) smaller than $n$. This is not the case whenever:

$$
K \mathrm{E}\left[\int_{0}^{1}[W(s)]^{2} d s\right]=K \int_{0}^{1} \mathrm{E}\left[[W(s)]^{2}\right] d s=K \int_{0}^{1} s \quad d s=\frac{K}{2} \geq n,
$$

which is a very restrictive condition.

Unit root $x_{k, t}$ processes induce unit root $Y_{t}$ processes. However, when applying the WAR(1) approach on series of realized covariance matrices of stock or currency returns, this assumption is not very realistic. Nevertheless, the estimation results based on this type of data reveal that estimated Wishart d.f. smaller than the dimension of the process are always accompanied by the fact that we cannot reject at $5 \%$ that the rank of matrix $I_{n}-M$ is smaller than $n$. Denote $H=I_{n}-M$. In the vector autoregressive framework, this result indicates that the latent autoregressive structure $x_{k, t}$ might be cointegrated with the following vector error correction representation:

$$
\Delta x_{k, t}=-H x_{k, t-1}+\varepsilon_{k, t} \quad \varepsilon_{k, t} \stackrel{\text { iid }}{\sim} \mathrm{N}(0, \Sigma)
$$

where $\operatorname{rank}(H)=r<n$. Proposition 3.3 states the asymptotic properties of the Wishart d.f. estimator under the assumption that the underlying latent autoregressive structure is cointegrated.

Proposition 3.3: Let $x_{k t}$ be cointegrated of rank $r$ as given in Equation (7). Then the the MM estimator of the Wishart d.f. is inconsistent and:

$$
\hat{K} \stackrel{d}{\rightarrow} K \int_{0}^{1}[W(s)]^{2} d s
$$

where $W(\cdot)$ stands for the standard Brownian motion.

Proof. See Appendix A.3.

Similar to the general non-stationary case, under cointegration assumptions, the estimated Wishart d.f. converge to values smaller than the true value, and often 
smaller than the dimension of the process. Although arguable in practice, these assumptions offer an accurate theoretical framework for describing why the Wishart d.f. are often estimated to be smaller than the dimension of the process. Complementarily, the next section discusses the result of estimating Wishart d.f. smaller than the dimension of the covariance process from the empirical perspective of using data which features distributional properties different from the ones required by the model.

\section{Empirical properties of WAR(1)}

In real terms, the WAR(1) model is estimated directly from a series of observed realized covariance matrices $\left(Y_{1}, \ldots, Y_{T}\right)$, which generally exhibits stationarity. Given that $\mathrm{E}\left[Y_{t}\right]=\Sigma^{*}(\infty)$, the MM estimator from Equation (3) can be derived from the empirical moments of $Y_{t}$ as follows:

$$
\hat{K}_{M M}(\alpha)=\frac{2 \hat{E}\left[\alpha^{\prime} Y_{t} \alpha\right]^{2}}{\hat{V}\left[\alpha^{\prime} Y_{t} \alpha\right]}=\frac{2}{\left(\frac{\sqrt{\hat{V}\left[\alpha^{\prime} Y_{t} \alpha\right]}}{\hat{E}\left[\alpha^{\prime} Y_{t} \alpha\right]}\right)^{2}}=\frac{2}{\hat{c}_{v}^{2}\left(\alpha^{\prime} Y_{t} \alpha\right)}
$$

where $c_{v}$ states for coefficient of variation. To estimate non-degenerate $\operatorname{WAR}(1)$ processes on series of covariance matrices of dimension $n \times n$, the following condition should hold: $\frac{2}{\hat{c}_{v}^{2}\left(\alpha^{\prime} Y_{t} \alpha\right)}>n$. Thus for $n=3, \hat{c}_{v}\left(\alpha^{\prime} Y_{t} \alpha\right)<0.81$, which is a very restrictive condition, especially for portfolios which include highly volatile stocks or especially during turbulent periods such as the previous financial crisis.

The empirical distribution of realized volatilities is better approximated by the log-normal (see Figure B. 1 and Taylor, 1996, Andersen, Bollerslev, Diebold and Ebens, 2001, Andersen, Bollerslev, Diebold and Labys, 2001, Bonato, Caporin and Ranaldo, 2009 among others) than by the Gamma distribution ${ }^{1}$. Consequently, the estimated Wishart d.f. inversely depend on the empirical variance of log-realized volatilities $^{2}$. This relationship is accentuated by the volatility clustering effect exhibited by the underlying series (Corsi, Kretschmer, Mittnik and Pigorsch, 2008).

During periods of relatively low volatility (see Table B. 1, panel C), the estimated d.f. are larger than the dimension of the process. This is generally common for short periods or calm financial periods. Similar results are reported by Gourieroux et al.

\footnotetext{
${ }^{1}$ Bonato (2009) shows that if $Y_{t}$ of dimension $n \times n$ is Wishart distributed with parameters $K, M$ and $\Sigma$, then for any vector $\alpha$ of dimension $n \times 1, \alpha^{\prime} Y_{t} \alpha \sim G a\left(\frac{K}{2}, 2 \alpha^{\prime} \Sigma(\infty) \alpha\right)$.

${ }^{2}$ The coefficient of variation of log-normal distributed variable $Y_{t}^{*}$ is given by $\sqrt{e^{V\left[\ln \left(Y_{t}^{*}\right)\right]}-1}$.
} 
(2009), who use one month of daily realized covariance matrices from October 1998. In favor of the low volatility argument are the results of Bonato et al. (2009), who estimate non-degenerate WAR(1) models on series of realized covariance matrices of U.S. treasury bond future and exchange rate returns, which usually exhibits lower volatility and variation in volatility than financial stocks do. However when applying the model to covariance matrices of stock returns during periods of large volatility (see Table B. 1, panels A and B), such as the previous financial crisis (see Table B. 1, panel B) the d.f. estimates are usually smaller than the dimension of the process, thus describing degenerate WAR(1) processes for the covarince matrix process.

\section{Concluding Remark}

In this note, we show that violations of the model assumptions lead to inconsistent estimators of Wishart d.f.. Latent autoregressive structures featuring cointegration instead of stationarity or portfolio volatility distributions closer to the log-normal than to the Gamma conduct to estimates of Wishart d.f, which are smaller than the dimension of the process, thus describing degenerate Wishart distributions for the underlying covariance matrices.

\section{Acknowledgement}

Financial support from the German Science Foundation within the project "Price-, Liquidity- and Credit Risks: Measurement and Allocation" is gratefully acknowledged. For helpful comments I would like to thank Winfried Pohlmeier and Valeri Voev.

\section{Appendix A: Proofs}

\section{A.1. Proof of Proposition 3.1}

Given that $\mathrm{E}\left[x_{k, t}\right]=0$, then we get for the process $Y_{t}$ in Equation (2) that:

$$
\mathrm{E}\left[Y_{t}\right]=\mathrm{E}\left[\sum_{k=1}^{K} x_{k, t} x_{k, t}^{\prime}\right]=\sum_{k=1}^{K} \mathrm{E}\left[x_{k, t} x_{k, t}^{\prime}\right]=\sum_{k=1}^{K} \mathrm{~V}\left[x_{k, t}\right]
$$


Denote $\mathrm{V}\left[x_{k, t}\right] \equiv \Sigma(\infty)$. Then $x_{k, t} \underset{\text { iid }}{\sim} \mathrm{N}(0, \Sigma(\infty))$. Let $\alpha$ be a vector of dimension $(n \times 1)$. Then $x_{k, t} \sim$ iid $\mathrm{N}\left(0, \alpha \Sigma(\infty) \alpha^{\prime}\right)$.

$$
\begin{gathered}
\mathrm{E}\left[Y_{t}\right]=K \Sigma(\infty) \equiv \Sigma^{*}(\infty) \\
\mathrm{V}\left[\alpha^{\prime} Y_{t} \alpha\right]=\mathrm{V}\left[\alpha^{\prime} \sum_{k=1}^{K} x_{k, t} x_{k, t}^{\prime} \alpha\right]=\sum_{k=1}^{K} \mathrm{~V}\left[\alpha^{\prime} x_{k, t} x_{k, t}^{\prime} \alpha\right]=K \mathrm{~V}\left[\alpha^{\prime} x_{k, t} x_{k, t}^{\prime} \alpha\right]=K \mathrm{~V}\left[\left(\alpha^{\prime} x_{k, t}\right)^{2}\right] \\
=K\left[\mathrm{E}\left[\left(\alpha^{\prime} x_{k, t}\right)^{4}\right]-\mathrm{E}\left[\left(\alpha^{\prime} x_{k, t}\right)^{2}\right]^{2}\right]=3 K\left(\alpha^{\prime} \mathrm{V}\left[x_{k, t}\right] \alpha\right)^{2}-K\left(\alpha^{\prime} \mathrm{V}\left[x_{k, t}\right] \alpha\right)^{2} \\
=3 K\left[\alpha^{\prime} \Sigma(\infty) \alpha\right]^{2}-K\left[\alpha^{\prime} \Sigma(\infty) \alpha\right]^{2}=2 K\left[\alpha^{\prime} \Sigma(\infty) \alpha\right]^{2}=\frac{2\left[\alpha^{\prime} \Sigma^{*}(\infty) \alpha\right]^{2}}{K}
\end{gathered}
$$

From Equation (A.3), we derive $K=\frac{2\left[\alpha^{\prime} \Sigma^{*}(\infty) \alpha\right]^{2}}{\mathrm{~V}\left[\alpha^{\prime} Y_{t} \alpha\right]}$, which leads to the MM estimator of $K$ from Equation (3).

$$
\begin{aligned}
K & =\frac{2\left[\alpha^{\prime} \Sigma^{*}(\infty) \alpha\right]^{2}}{\mathrm{~V}\left[\alpha^{\prime} Y_{t} \alpha\right]}=\frac{2\left(\mathrm{E}\left[\alpha^{\prime} Y_{t} \alpha\right]\right)^{2}}{\mathrm{~V}\left[\alpha^{\prime} Y_{t} \alpha\right]}=\frac{2\left(\mathrm{E}\left[\alpha^{\prime} Y_{t} \alpha\right]\right)^{2}}{\mathrm{~V}\left[\alpha^{\prime} Y_{t} \alpha\right]}=\frac{\left(\alpha^{\prime} \mathrm{E}\left[Y_{t}\right] \alpha\right)^{2}}{K\left(\alpha^{\prime} \Sigma(\infty) \alpha\right)^{2}} \\
& =\frac{\left(\alpha^{\prime} \mathrm{E}\left[Y_{t}\right] \alpha\right)^{2}}{\left(\alpha^{\prime} \Sigma(\infty) \alpha\right)\left(K \alpha^{\prime} \Sigma(\infty) \alpha\right)}=\frac{\left(\alpha^{\prime} \mathrm{E}\left[Y_{t}\right] \alpha\right)^{2}}{\left(\alpha^{\prime} \Sigma(\infty) \alpha\right)\left(\alpha^{\prime} \mathrm{E}\left[Y_{t}\right] \alpha\right)}=\frac{\alpha^{\prime} \mathrm{E}\left[Y_{t}\right] \alpha}{\alpha^{\prime} \Sigma(\infty) \alpha}
\end{aligned}
$$

From Equation (A.4) we can derive $K$ as follows:

$$
K=\frac{\alpha^{\prime} \mathrm{E}\left[Y_{t}\right] \alpha}{\alpha^{\prime} \Sigma(\infty) \alpha}=\frac{\mathrm{E}\left[\sum_{k=1}^{K}\left(\alpha^{\prime} x_{k, t}\right)^{2}\right]}{\alpha^{\prime} \Sigma(\infty) \alpha}=\mathrm{E}\left[\sum_{k=1}^{K} \frac{\left(\alpha^{\prime} x_{k, t}\right)^{2}}{\alpha^{\prime} \Sigma(\infty) \alpha}\right] \equiv \mathrm{E}\left[\sum_{k=1}^{K} z_{k, t}^{2}\right] \equiv \mathrm{E}\left[S_{t}\right]
$$

where $z_{k, t} \equiv \frac{\alpha^{\prime} x_{k, t}}{\sqrt{\alpha^{\prime} \Sigma(\infty) \alpha}} \stackrel{\text { iid }}{\sim} \mathrm{N}(0,1)$ and $S_{t} \equiv \sum_{k=1}^{K} z_{k, t}^{2} \sim \chi_{(K)}$. In this format, the MM estimator from Equation (3) can be written as:

$$
\hat{K}=\frac{\sum_{t=1}^{T} S_{t}}{T}=\overline{S_{t}}
$$

Under stationarity conditions (i.e., eigenvalues of $M$ are strictly smaller than 1 ), $\mathrm{V}\left[x_{k, t+1}\right]=$ $M \mathrm{~V}\left[x_{k, t}\right] M^{\prime}+\mathrm{V}\left[\varepsilon_{k, t+1}\right]$, which implies that $\Sigma(\infty)=M \Sigma(\infty) M^{\prime}+\Sigma$ and $\Sigma^{*}(\infty)=$ $M \Sigma^{*}(\infty) M^{\prime}+\Sigma^{*}$, with $\Sigma^{*}=K \Sigma$. Moreover, stationary $x_{k, t}$ processes implies that $S_{t}$ is a stochastic stationary process. Given that $x_{k, t}$ are serially correlated, $S_{t}$ are also serially correlated. Therefore, in order to derive the distribution of $\overline{S_{t}}$ (i.e. $\hat{K}$ ) we apply the Central Limit Theorem (CLT) for Stationary Stochastic Processes (see Hamilton (1994), pp.195), which states that:

$$
\sqrt{T}\left(\overline{S_{t}}-\mathrm{E}\left[S_{t}\right]\right) \stackrel{d}{\rightarrow} N\left(0, \sum_{j=-\infty}^{\infty} \gamma_{j}\right)
$$


where $\gamma_{j}=\mathrm{E}\left[\left(S_{t}-\mathrm{E}\left[S_{t}\right]\right)\left(S_{t-j}-\mathrm{E}\left[S_{t}\right]\right)\right]$ with $\mathrm{E}\left[S_{t}\right]=K$ for all $t$. From Equation (A.6), we deduce that:

$$
\sqrt{T}(\hat{K}-K) \stackrel{d}{\rightarrow} N\left(0, \sum_{j=-\infty}^{\infty} \gamma_{j}\right)
$$

In Equation (A.5), $\hat{K}$ is consistent, but not efficient (depends on the choice of $\alpha$ ). However, for our purposes the consistency is relevant, and not the efficiency of the estimator.

\section{A.2. Proof of Proposition 3.2}

Let $x_{k t}$ have a unit root and be written as:

$$
x_{k, t}=x_{k, t-1}+\varepsilon_{k, t} \quad \varepsilon_{k, t} \stackrel{\text { iid }}{\sim} \mathrm{N}(0, \Sigma)
$$

Then $x_{k, t}$ can be recursively written as follows:

$$
x_{k, t}=\varepsilon_{k, t}+\varepsilon_{k, t-1}+\ldots+\varepsilon_{k, 1}
$$

and $\mathrm{V}\left[x_{k, t}\right] \equiv \Sigma(\infty)=t \Sigma$. Expression (A.4) becomes:

$$
K=\frac{\alpha^{\prime} \mathrm{E}\left[Y_{t}\right] \alpha}{t \alpha^{\prime} \Sigma \alpha}=\frac{\mathrm{E}\left[\sum_{k=1}^{K}\left(\alpha^{\prime} x_{k, t}\right)^{2}\right]}{t \alpha^{\prime} \Sigma \alpha}=\frac{\mathrm{E}\left[\sum_{k=1}^{K}\left(\frac{\alpha^{\prime} x_{k, t}}{\sqrt{\alpha^{\prime} \Sigma \alpha}}\right)^{2}\right]}{t} \equiv \frac{\mathrm{E}\left[\sum_{k=1}^{K}\left(z_{k, t}^{*}\right)^{2}\right]}{t},
$$

where $z_{k, t}^{*} \equiv \frac{\alpha^{\prime} x_{k, t}}{\sqrt{\alpha^{\prime} \Sigma \alpha}}=z_{k, t-1}^{*}+\varepsilon_{k, t}^{*}$, with $\varepsilon_{k, t}^{*} \stackrel{\text { iid }}{\sim} \mathrm{N}(0,1)$. The MM estimator of $K$ becomes:

$$
\hat{K}=\frac{\sum_{t=1}^{T} \sum_{k=1}^{K}\left(z_{k, t}^{*}\right)^{2}}{T^{2}}
$$

Given that $z_{k, t}^{*}$ follows a random walk, for which Hamilton (1994) shows that (Proposition 17.1, point(e)):

$$
T^{-2} \sum_{t=1}^{T}\left(z_{k, t}^{*}\right)^{2} \rightarrow \int_{0}^{1}[W(s)]^{2} d s,
$$

where $W(\cdot)$ stands for the standard Brownian motion, we derive the the asymptotic distribution of $\hat{K}$ to be:

$$
\hat{K} \stackrel{d}{\rightarrow} K \int_{0}^{1}[W(s)]^{2} d s
$$


Consequently the estimator of the Wishart d.f. derived under unit root assumptions, converges in distribution to a random variable with expectation smaller than $K$ :

$$
\mathrm{E}\left[K \int_{0}^{1}[W(s)]^{2} d s\right]=K \int_{0}^{1} \mathrm{E}\left[[W(s)]^{2}\right] d s=K \int_{0}^{1} s \quad d s=\frac{K}{2}<K
$$

Similar to the stationary case, the estimator of $K$ from Equation (A.11) is not efficient. However, Equation (A.14) shows that it is also downward biased.

\section{A.3. Proof of Proposition 3.3}

The process $x_{k, t}$ in Equation (7) can be written as:

$$
x_{k, t}=\left(I_{n}-H\right) x_{k, t-1}+\varepsilon_{k, t},
$$

Because $\operatorname{rank}(H)=r<n$, than matrix $H$ can be written as: $H=B \Gamma^{\prime}$, with $B$ and $\Gamma$ be of dimension $n \times r$ and of full column rank. Then exists a non-zero matrix $\mathrm{C}$ of dimension $n \times r$ such that $C^{\prime} B=0_{(r, r)}$ i.e., $\mathrm{C}$ is orthogonal to B. Pre-multiplying Equation (A.15) by $C^{\prime}$, we get:

$$
\begin{aligned}
u_{k, t} & \equiv C^{\prime} x_{k, t}=C^{\prime}\left(I_{n}-H\right) x_{k, t-1}+C^{\prime} \varepsilon_{k, t}=C^{\prime} x_{k, t-1}-C^{\prime} H x_{k, t-1}+C^{\prime} \varepsilon_{k, t} \\
& =C^{\prime} x_{k, t-1}-C^{\prime} B \Gamma^{\prime} x_{k, t-1}+C^{\prime} \varepsilon_{k, t}=C^{\prime} x_{k, t-1}+C^{\prime} \varepsilon_{k, t}=u_{k, t-1}+\eta_{k, t}
\end{aligned}
$$

where $\eta_{k, t} \sim \operatorname{iid} \sim \mathrm{N}\left(0, C^{\prime} \Sigma C\right)$. Denote $C^{\prime} \Sigma C \equiv \Omega$. The process $u_{k, t}$ is a random walk, for which $\mathrm{V}\left[u_{k, t}\right]=C^{\prime} \mathrm{V}\left[x_{k, t}\right] C=C^{\prime} \Sigma(\infty) C=t \Omega$.

By multiplying both sides of Equation (A.2) by $\mathrm{C}$ and respectively $\mathrm{C}^{\prime}$, we get:

$$
\mathrm{E}\left[C^{\prime} Y_{t} C\right]=K C^{\prime} \Sigma(\infty) C=K t \Omega
$$

Similar to Equation (A.4), we derive $K$ to be given by:

$$
\begin{aligned}
K & =\frac{\alpha^{\prime} \mathrm{E}\left[C^{\prime} Y_{t} C\right] \alpha}{t \alpha^{\prime} \Omega \alpha}=\frac{\mathrm{E}\left[\sum_{k=1}^{K}\left(\alpha^{\prime} C^{\prime} x_{k, t}\right)^{2}\right]}{t \alpha^{\prime} \Omega \alpha}=\frac{\mathrm{E}\left[\sum_{k=1}^{K}\left(\alpha^{\prime} u_{k, t}\right)^{2}\right]}{t \alpha^{\prime} \Omega \alpha} \\
& =\frac{\mathrm{E}\left[\sum_{k=1}^{K}\left(\frac{\alpha^{\prime} u_{k, t}}{\sqrt{\alpha^{\prime} \Omega \alpha^{\prime}}}\right)^{2}\right]}{t} \equiv \frac{\mathrm{E}\left[\sum_{k=1}^{K}\left(u_{k, t}^{*}\right)^{2}\right]}{t},
\end{aligned}
$$

where $u_{k, t}^{*} \equiv \frac{\alpha^{\prime} u_{k, t}}{\sqrt{\alpha^{\prime} \Omega \alpha}}=u_{k, t-1}^{*}+\eta_{k, t}^{*}$, with $\eta_{k, t}^{*} \stackrel{\text { iid }}{\sim} \mathrm{N}(0,1)$. The MM estimator of $K$ becomes:

$$
\hat{K}=\frac{\sum_{t=1}^{T} \sum_{k=1}^{K}\left(u_{k, t}^{*}\right)^{2}}{T^{2}} .
$$


Similar to the unit root case, we derive the asymptotic distribution of $\hat{K}$ to be:

$$
\hat{K} \stackrel{d}{\rightarrow} K \int_{0}^{1}[W(s)]^{2} d s .
$$




\section{Appendix B: Tables and Figures}

Table B. 1: Estimation results of WAR(1) model on different time samples, $n=3$. All $\hat{K}$ are significant at $1 \%$. The table reports the Maximum Likelihood estimates (Bonato, 2009) and the MM estimates of $K$, computed from observed series of daily realized covariance matrices of stock returns of Citigroup (C), General Electric (GE) and Home Depot Inc. (HD) traded on NYSE, for different portfolio allocations: $\alpha=(1,0,0)^{\prime}, \alpha=(0,1,0)^{\prime}, \alpha=(0,0,1)^{\prime}$ and $\alpha=(1,1,1)^{\prime}$ and for different estimation windows: 01.01.2001-30.07.2008 (period 1), 17.07.2007-30.07.2008 (period 2) and 01.05.2006-31.05.2006 (period 3). The daily realized covariance matrices are computed from 5-minute mid-quote returns by following the procedure described in Andersen, Bollerslev, Diebold and Ebens (2001). Standard errors are reported in parentheses.

\begin{tabular}{|c|c|c|c|c|}
\hline$\alpha$ & $\hat{K}_{M M}$ & $\hat{K}_{M L}$ & $\hat{V}\left[\ln \left(\alpha^{\prime} Y_{t} \alpha\right)\right]$ & $\hat{c}_{v}\left(\alpha^{\prime} Y_{t} \alpha\right)$ \\
\hline \multicolumn{5}{|c|}{ Panel A: Period 1 } \\
\hline \hline$(1,0,0)^{\prime}$ & $0.615(0.132)$ & $0.853(0.012)$ & 1.216 & 1.819 \\
$(0,1,0)^{\prime}$ & $0.964(0.136)$ & $1.094(0.015)$ & 0.935 & 1.432 \\
$(0,0,1)^{\prime}$ & $1.335(0.130)$ & $1.381(0.017)$ & 0.711 & 1.224 \\
$(1,1,1)^{\prime}$ & $0.890(0.115)$ & $1.031(0.014)$ & 0.988 & 1.505 \\
\hline \multicolumn{5}{|c|}{ Panel B: Period 2 } \\
\hline \hline$(1,0,0)^{\prime}$ & $2.102(0.303)$ & $1.417(0.047)$ & 0.815 & 1.012 \\
$(0,1,0)^{\prime}$ & $2.686(0.494)$ & $2.111(0.059)$ & 0.472 & 0.878 \\
$(0,0,1)^{\prime}$ & $2.811(0.733)$ & $2.161(0.059)$ & 0.477 & 0.861 \\
$(1,1,1)^{\prime}$ & $2.352(0.406)$ & $1.645(0.051)$ & 0.664 & 0.955 \\
\hline \multicolumn{5}{|c|}{ Panel C: Period 3 } \\
\hline \hline$(1,0,0)^{\prime}$ & $10.330(3.551)$ & $6.474(0.365)$ & 0.158 & 0.428 \\
$(0,1,0)^{\prime}$ & $7.403(3.710)$ & $3.765(0.247)$ & 0.308 & 0.527 \\
$(0,0,1)^{\prime}$ & $6.233(3.769)$ & $3.700(0.271)$ & 0.277 & 0.587 \\
$(1,1,1)^{\prime}$ & $6.491(2.277)$ & $3.510(0.264)$ & 0.315 & 0.569 \\
\hline
\end{tabular}


alpha $=(1,0,0)^{\prime}$
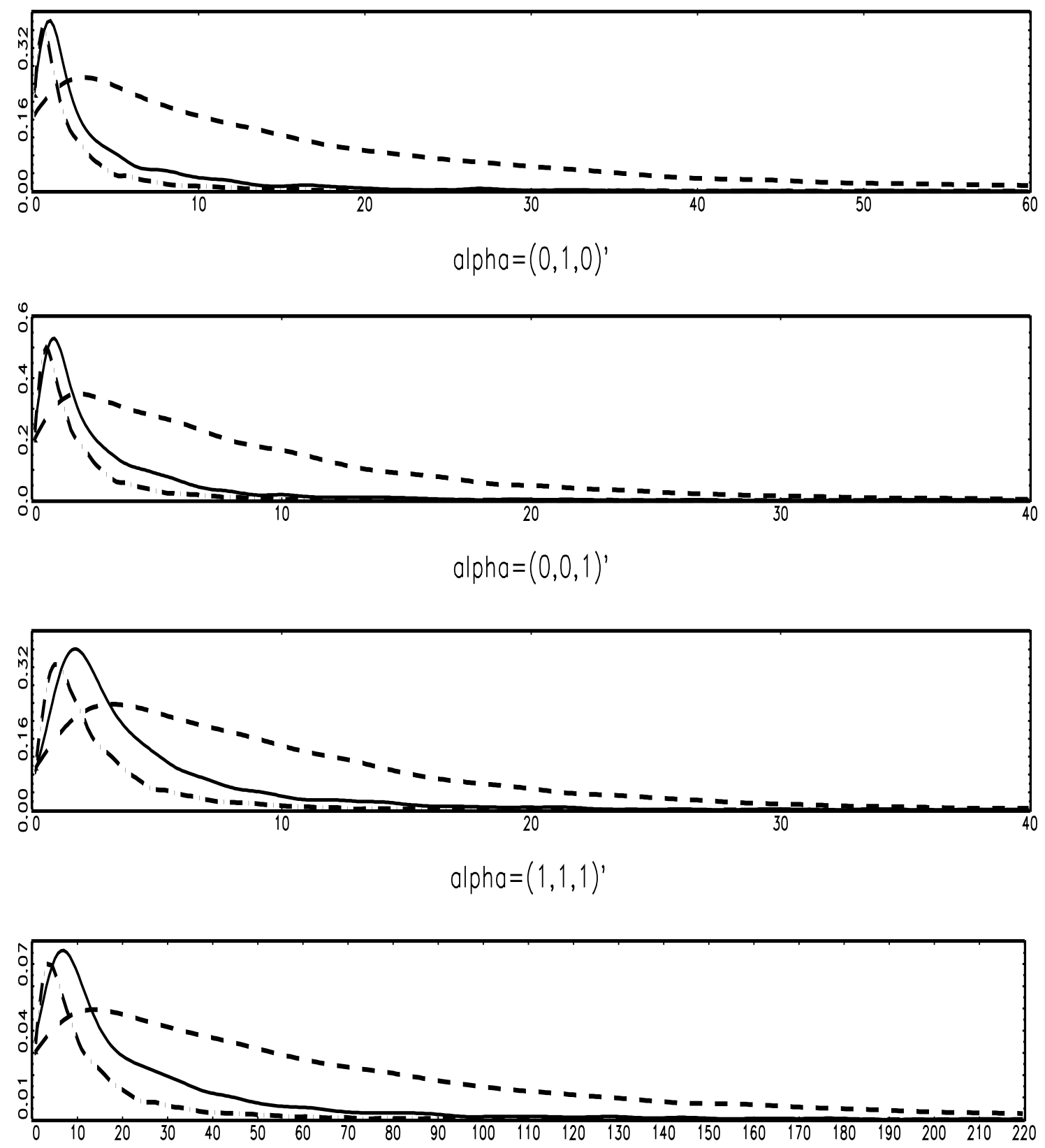

Figure B. 1: Kernel densities of portfolio realized volatilities. Stocks considered: Citigroup (C), General Electric (GE) and Home Depot Inc. (HD) traded on NYSE from 01.01.2001 until 30.07.2008. Dashed lines correspond to the fitted Gamma distributions (Bonato, 2009), dotted and dashed lines correspond to fitted log-normal distributions and solid lines are the kernel densities of the underlying series; first (upper) panel: $\alpha=(1,0,0)^{\prime}$; second panel: $\alpha=(0,1,0)^{\prime}$ : third panel: $\alpha=(0,0,1)^{\prime}$ and fourth (lower) panel: $\alpha=(1,1,1)^{\prime}$. 


\section{References}

Andersen, T. G., T. Bollerslev, F. X. Diebold, and H. Ebens (2001): "The Distribution of Stock Return Volatility," Journal of Financial Economics, $61,43-76$.

Andersen, T. G., T. Bollerslev, F. X. Diebold, and P. Labys (2001): "The Distribution of Exchange Rate Volatility," Journal of the American Statistical Association, 96, 42-55.

Bonato, M. (2009): "Estimating the Degrees of Freedom of the Realized Volatility Wishart Autoregressive Model," Working paper.

Bonato, M., M. Caporin, and A. Ranaldo (2009): "Forecasting Realized (Co)variances with a Block Wishart Autoregressive Model," Working paper.

Corsi, F., U. Kretschmer, S. Mittnik, and C. Pigorsch (2008): "Volatility of Realized Volatility," Econometric Reviews, 27, 46-78.

Gourieroux, C., J. Jasiak, and R. Sufana (2009): "The Wishart Autoregressive Process of Multivariate Stochastic Volatility," Journal of Econometrics, 150, $167-181$.

Hamilton, J. D. (1994): Time Series Analysis. Princeton University Press, Princeton, New Jersey.

TAYlor, S. J. (1986): Modelling Financial Time Series. Wiley, New York. 